\title{
Choosing OWA operator weights in the field of Social Choice
}

\author{
Bonifacio Llamazares * \\ Dep. de Economía Aplicada, PRESAD Research Group, Universidad de \\ Valladolid, Avda. Valle de Esgueva 6, 47011 Valladolid, Spain.
}

\begin{abstract}
One of the most important issues in the theory of OWA operators is the determination of associated weights. This matter is essential in order to use the best-suited OWA operator in each aggregation process. Given that some aggregation processes can be seen as extensions of majority rules to the field of gradual preferences, it is possible to determine the OWA operator weights by taking into account the class of majority rule that we want to obtain when individuals do not grade their pairwise preferences. However, a difficulty with this approach is that the same majority rule can be obtained through a wide variety of OWA operators. For this reason, a model for selecting the best-suited OWA operators is proposed in this paper.
\end{abstract}

Key words: OWA operators, Aggregation operator weights, Majority rules.

\section{Introduction}

Ordered weighted averaging (OWA) operators were introduced by Yager [29] as a tool for information aggregation. An OWA operator is similar to a weighted mean, but with the values of the variables previously ordered in a decreasing way. Thus, contrary to the weighted means, the weights are not associated with concrete variables. Consequently, OWA operators satisfy symmetry. Moreover, OWA operators generalize the arithmetic mean and the median, and they also exhibit some other interesting properties such as monotonicity, idempotence, and compensativeness (i.e., the value of an OWA operator is located between the minimum and the maximum values of the variables). Because of these

* Tel.: +34-983-186544; fax: +34-983-423299.

Email address: boni@eco.uva.es (Bonifacio Llamazares). 
properties, OWA operators have been widely used in the literature (see, for instance, Yager and Kacprzyk [36] and Calvo, Mayor and Mesiar [2]).

One of the most important issues in the field of OWA operators is the determination of the weights of the OWA operator. This matter is different from that raised in the framework of weighted means, where the weights are associated with concrete variables and as such they reflect the importance of experts.

In the field of OWA operators, one of the first approaches, suggested by O'Hagan [18], lies in selecting the vector that maximizes the entropy of the OWA weights for a given level of orness. This methodology has also been used by Fullér and Majlender [4]. They transfer the constrained optimization problem to a polynomial equation, which is solved for determining the optimal weighting vector. To avoid the resolution of a nonlinear optimization problem, Yager [32] introduces a simpler procedure that tries to keep the spirit of maximizing the entropy for a given level of orness. Similar approaches (through a fixed orness level) have also been proposed by Fullér and Majlender [5], Wang and Parkan [21], Majlender [15], and Amin and Emrouznejad [1]. The first authors suggest selecting the vector that minimizes the variance of the weighting vector in order to obtain the minimal variability OWA weights, while the second cited authors propose minimizing the disparities between adjacent weights. This model has been extended by Amin and Emrouznejad [1] by minimizing the maximum disparity of any pair of weights. On the other hand, Majlender [15] determines a parametric class of OWA operators having maximal Rényi entropy OWA weights. Other methods to determine the OWA operators weights have been suggested by Yager [29-31,34], Filev and Yager [3], Yager and Filev [35], Nettleton and Torra [17], Xu and Da [27], Liu and Chen [10], Xu [25,26] and Liu [9]. A survey of the main methods for determining OWA operator weights can be found in $\mathrm{Xu}$ [25].

The approach presented in this paper is different from those of the previous authors. In our case we want to determine the OWA operator weights that allow us to extend, through the OWA operator, some classes of majority rules obtained when individuals do not grade their preferences between two alternatives (simple, Pareto, absolute special majorities, etc.). In the papers referenced earlier $[18,4,5,21,15,1]$, once the level of orness is fixed, the weights are determined through properties that only concern them (to maximize the entropy, to minimize the variance, etc.). Therefore, the aggregation procedure is only taken into account when we fix the level of orness. However, in our case we focus on the performance of the aggregation operator when individuals do not grade their preferences between the alternatives. On the other hand, in order to guarantee an egalitarian treatment of the alternatives, we have considered self-dual OWA operators, whose level of orness is 0.5 . In this case, the methodologies based on fixing a level of orness always have the same solution: the arithmetic mean. 
It is important to note that self-dual OWA operators have been considered by $\mathrm{Xu}$ [25] and Yager [34], among others (for the representation and construction of self-dual aggregation operators, see Maes, Saminger and De Baets [14]). $\mathrm{Xu}$ [25] introduces a procedure for obtaining OWA weights through Gaussian distribution, while Yager [34] generalizes Xu's idea by means of a new family of OWA operators: centered OWA operators. In this model, the problem is how to choose a specific OWA operator of this class and, in Xu's method, how to justify the choice of that weighting vector.

Different ways to deal with fuzzy opinions in group decision making exist in recent literature (see, for instance, Xu [24], Yager [33], García-Lapresta [6], Liu and Lou [11], Pasi and Yager [19], Peláez and Doña [20], and Wang and Parkan $[22,23])$. The procedure developed in this paper to choose an alternative from individual preferences is as follows: Once preferences on a pair of alternatives have been shown by the individuals through values between 0 and 1, we obtain the collective intensity of preference by means of an aggregation operator. From this value and through a kind of strong $\alpha$-cut, where $\alpha \in\left[\frac{1}{2}, 1\right)$, we can decide if an alternative is chosen or if both alternatives are collectively indifferent. When individuals do not grade their preferences, that is, when they are represented through the values $0,1 / 2$, and 1 , the previous procedure allows us to obtain a majority rule. Hence, once $\alpha$ fixed, it is possible to know what class of majority rule is present in the aggregation process according to the used operator.

We note that this procedure has already been used to characterize some classes of aggregation functions that extend some well-known majority rules. Thus, García-Lapresta and Llamazares [8] generalize two classes of majorities based on difference of votes by using quasiarithmetic means and window OWA operators as aggregation functions. Likewise, Llamazares [12] has characterized the OWA operators that generalize simple and absolute special majorities.

In this paper we characterize the OWA operators that allow us to extend Pareto majority. On the other hand, since we can obtain the same majority rule by means of a wide variety of OWA operators, we propose a method to determine the best-suited OWA operators in order to extend simple, Pareto, and absolute special majorities.

The organization of the paper is as follows: In Section 2, we introduce basic concepts and some known results of OWA operators. We also show the OWA operators that generalize simple and absolute special majorities. In Section 3 , we characterize the OWA operators that generalize Pareto majority. The technical Section 4 is necessary for Section 5, where we propose a model to obtain the best-suited OWA operators to generalize some classes of majority rules. We present some conclusions in Section 6. 


\section{Preliminaries}

We consider $m$ voters, with $m \geq 3$, and two alternatives $x$ and $y$. Voters represent their preferences between $x$ and $y$ through variables $r_{i}$. If the individuals grade their preferences, then $r_{i} \in[0,1]$ denotes the intensity with which voter $i$ prefers $x$ to $y$. We also suppose that $1-r_{i}$ is the intensity with which voter $i$ prefers $y$ to $x$. If the individuals do not grade their preferences, then $r_{i} \in\left\{0, \frac{1}{2}, 1\right\}$ represents that voter $i$ prefers $x$ to $y\left(r_{i}=1\right)$, prefers $y$ to $x\left(r_{i}=0\right)$, or is indifferent between both alternatives $\left(r_{i}=\frac{1}{2}\right)$. The justification of this three-valued representation can be found in García-Lapresta and Llamazares [7].

A profile of preferences is a vector $\mathbf{r}=\left(r_{1}, \ldots, r_{m}\right)$ that describes voters' preferences between alternative $x$ and alternative $y$. Obviously, $\mathbf{1}-\mathbf{r}=(1-$ $\left.r_{1}, \ldots, 1-r_{m}\right)$ shows voters' preferences between $y$ and $x$. For each profile of preferences, the collective preference will be obtained by means of an aggregation function.

\subsection{Aggregation functions}

Definition 1 An aggregation function is a mapping $F:[0,1]^{m} \longrightarrow[0,1]$. A discrete aggregation function $(D A F)$ is a mapping $H:\left\{0, \frac{1}{2}, 1\right\}^{m} \longrightarrow\left\{0, \frac{1}{2}, 1\right\}$.

The interpretation of collective preference is consistent with the foregoing interpretation for individual preferences. Thus, if $F$ is an aggregation function, then $F(\mathbf{r})$ is the intensity with which $x$ is collectively preferred to $y$. When $H$ is a DAF, then $H(\mathbf{r})$ shows us if an alternative is collectively preferred to the other $(H(\mathbf{r}) \in\{0,1\})$, or the alternatives are collectively indifferent $\left(H(\mathbf{r})=\frac{1}{2}\right)$.

Next we present some well-known properties of aggregation functions: Symmetry, monotonicity, strict monotonicity, and self-duality. Symmetry means that collective intensity of preference depends on only the set of individual intensities of preference, but not on which individuals have these preferences. Monotonicity means that collective intensity of preference does not decrease if no individual intensity decreases. Strict monotonicity means that collective intensity of preference increases if some individual intensity increases. Finally, self-duality means that if everyone reverses their preferences between $x$ and $y$, then the collective preference is also reversed. Given $\mathbf{r}, \mathbf{s} \in[0,1]^{m}$ and $\sigma$ a permutation on $\{1, \ldots, m\}$, we will use the following notation: $\mathbf{r}_{\sigma}=$ $\left(r_{\sigma(1)}, \ldots, r_{\sigma(m)}\right) ; \mathbf{1}=(1, \ldots, 1) ; \mathbf{r} \geq \mathbf{s}$ will denote $r_{i} \geq s_{i}$ for all $i \in\{1, \ldots, m\}$; and $\mathbf{r}>\mathbf{s}$ will mean $\mathbf{r} \geq \mathbf{s}$ and $\mathbf{r} \neq \mathbf{s}$. 
Definition 2 Let $F$ be an aggregation function.

(1) $F$ is symmetric if for all profile $\mathbf{r} \in[0,1]^{m}$ and for all permutation $\sigma$ of $\{1, \ldots, m\}$ the following holds

$$
F\left(\mathbf{r}_{\sigma}\right)=F(\mathbf{r})
$$

(2) $F$ is monotonic if for all pair of profiles $\mathbf{r}, \mathbf{s} \in[0,1]^{m}$ the following holds

$$
\mathbf{r} \geq \mathbf{s} \Rightarrow F(\mathbf{r}) \geq F(\mathbf{s}) .
$$

(3) $F$ is strictly monotonic if for all pair of profiles $\mathbf{r}, \mathbf{s} \in[0,1]^{m}$ the following holds

$$
\mathbf{r}>\mathbf{s} \Rightarrow F(\mathbf{r})>F(\mathbf{s})
$$

(4) $F$ is self-dual if for all profile $\mathbf{r} \in[0,1]^{m}$ the following holds

$$
F(\mathbf{1}-\mathbf{r})=1-F(\mathbf{r}) .
$$

All the previous properties, except strict monotonicity, are also valid for DAFs. Next we show some consequences of the previous properties. The cardinal of a set will be denoted by \#.

Remark 3 If $H$ is a symmetric DAF, then $H(\mathbf{r})$ depends on only the number of $1, \frac{1}{2}$, and 0 . Given a profile $\mathbf{r}$, if we consider:

$$
m_{1}=\#\left\{i \mid r_{i}=1\right\}, \quad m_{2}=\#\left\{i \mid r_{i}=\frac{1}{2}\right\}, \quad m_{3}=\#\left\{i \mid r_{i}=0\right\},
$$

then $m_{1}+m_{2}+m_{3}=m$.

Definition 4 Let $H$ be a symmetric DAF and

$$
\mathcal{M}=\left\{\left(m_{1}, m_{2}, m_{3}\right) \in\{0, \ldots, m\}^{3} \mid m_{1}+m_{2}+m_{3}=m\right\} .
$$

We say that $H$ is represented by $h: \mathcal{M} \longrightarrow\left\{0, \frac{1}{2}, 1\right\}$, defined by

$$
h\left(m_{1}, m_{2}, m_{3}\right)=H\left(1, \stackrel{\left(m_{1}\right)}{\cdot}, 1, \frac{1}{2}, \stackrel{\left(m_{2}\right)}{\cdot}, \frac{1}{2}, 0, \stackrel{\left(m_{3}\right)}{\cdot}, 0\right) .
$$

Definition 5 The binary relation $\succeq$ on $\mathcal{M}$ is defined by

$$
\left(m_{1}, m_{2}, m_{3}\right) \succeq\left(n_{1}, n_{2}, n_{3}\right) \Leftrightarrow\left\{\begin{array}{l}
m_{1} \geq n_{1} \\
\text { and } \\
m_{1}+m_{2} \geq n_{1}+n_{2}
\end{array}\right.
$$


We note that $\succeq$ is a partial order on $\mathcal{M}$ (reflexive, antisymmetric, and transitive binary relation).

Remark 6 If $H$ is a symmetric DAF represented by $h$, then it is monotonic if and only if $h\left(m_{1}, m_{2}, m_{3}\right) \geq h\left(n_{1}, n_{2}, n_{3}\right)$ for all $\left(m_{1}, m_{2}, m_{3}\right),\left(n_{1}, n_{2}, n_{3}\right) \in$ $\mathcal{M}$ such that $\left(m_{1}, m_{2}, m_{3}\right) \succeq\left(n_{1}, n_{2}, n_{3}\right)$.

Remark 7 If $H$ is a symmetric DAF represented by $h$, then it is self-dual if and only if $h\left(m_{3}, m_{2}, m_{1}\right)=1-h\left(m_{1}, m_{2}, m_{3}\right)$ for all $\left(m_{1}, m_{2}, m_{3}\right) \in \mathcal{M}$. In this case, $H$ is characterized by the set $h^{-1}(\{1\})$, since

$$
\begin{aligned}
& h^{-1}(\{0\})=\left\{\left(m_{1}, m_{2}, m_{3}\right) \in \mathcal{M} \mid h\left(m_{3}, m_{2}, m_{1}\right)=1\right\} \\
& h^{-1}\left(\left\{\frac{1}{2}\right\}\right)=\mathcal{M} \backslash\left(h^{-1}(\{1\}) \cup h^{-1}(\{0\})\right) .
\end{aligned}
$$

When a DAF is self-dual, both alternatives have an egalitarian treatment. Therefore, if the DAF is also symmetric and the number of voters who prefer $x$ to $y$ coincides with the number of voters who prefer $y$ to $x$, then $x$ and $y$ are collectively indifferent.

Remark 8 If $H$ is a symmetric and self-dual DAF represented by $h$, then $h\left(m_{1}, m_{2}, m_{3}\right)=\frac{1}{2}$ for all $\left(m_{1}, m_{2}, m_{3}\right) \in \mathcal{M}$ such that $m_{1}=m_{3}$.

By Remark 7 , it is possible to define a symmetric and self-dual DAF $H$ by means of the elements $\left(m_{1}, m_{2}, m_{3}\right) \in \mathcal{M}$ where the mapping that represents $H$ takes the value 1 . Based on this, we now show some DAFs widely used in real decisions.

\section{Definition 9}

(1) The simple majority, $H_{S}$, is the symmetric and self-dual DAF defined by

$$
h\left(m_{1}, m_{2}, m_{3}\right)=1 \Leftrightarrow m_{1}>m_{3} .
$$

(2) The absolute majority, $H_{A}$, is the symmetric and self-dual DAF defined by

$$
h\left(m_{1}, m_{2}, m_{3}\right)=1 \Leftrightarrow m_{1}>\frac{m}{2} .
$$

(3) The Pareto majority, $H_{P}$, is the symmetric and self-dual DAF defined by

$$
h\left(m_{1}, m_{2}, m_{3}\right)=1 \Leftrightarrow m_{1}>0 \text { and } m_{3}=0 .
$$

(4) The unanimous majority, $H_{U}$, is the symmetric and self-dual DAF defined by

$$
h\left(m_{1}, m_{2}, m_{3}\right)=1 \Leftrightarrow m_{1}=m .
$$


(5) Given $\beta \in\left[\frac{1}{2}, 1\right)$, the absolute special majority $Q_{\beta}$ is the symmetric and self-dual DAF defined by

$$
h\left(m_{1}, m_{2}, m_{3}\right)=1 \Leftrightarrow m_{1}>\beta m .
$$

It should be noted that absolute and unanimous majorities are specific cases of absolute special majorities.

Given an aggregation function, we can generate different DAFs by means of a parameter $\alpha \in\left[\frac{1}{2}, 1\right)$. The procedure employed is based on strong $\alpha$ cuts. Moreover, it is easy to check that the DAFs obtained are symmetric, monotonic, and self-dual when the original aggregation function satisfies these properties.

Definition 10 Let $F$ be an aggregation function and $\alpha \in\left[\frac{1}{2}, 1\right)$. Then the $\alpha-D A F$ associated with $F$ is the DAF $F_{\alpha}$ defined by

$$
F_{\alpha}(\mathbf{r})=\left\{\begin{array}{l}
1, \text { if } F(\mathbf{r})>\alpha, \\
\frac{1}{2}, \text { if } 1-\alpha \leq F(\mathbf{r}) \leq \alpha, \\
0, \text { if } F(\mathbf{r})<1-\alpha .
\end{array}\right.
$$

Remark 11 Given an aggregation function $F$ and $\alpha \in\left[\frac{1}{2}, 1\right)$, the following statements hold:

(1) If $F$ is symmetric, then $F_{\alpha}$ is also symmetric.

(2) If $F$ is monotonic, then $F_{\alpha}$ is also monotonic.

(3) If $F$ is self-dual, then $F_{\alpha}$ is also self-dual.

Similar to the case of symmetric DAFs, when $F$ is a symmetric aggregation function, the restriction $\left.F\right|_{\left\{0, \frac{1}{2}, 1\right\}^{m}}$ can be represented by $f: \mathcal{M} \longrightarrow[0,1]$, where

$$
f\left(m_{1}, m_{2}, m_{3}\right)=F\left(1, \stackrel{\left(m_{1}\right)}{\cdot}, 1, \frac{1}{2}, \stackrel{\left(m_{2}\right)}{\cdot}, \frac{1}{2}, 0, \stackrel{\left(m_{3}\right)}{\cdot}, 0\right) .
$$

Now we show the relationship between $f$ and the family of mappings $f_{\alpha}$ that represent the $\alpha$-DAFs associated with $F$.

Remark 12 Let $F$ be a symmetric aggregation function and $\alpha \in\left[\frac{1}{2}, 1\right)$. Then $F_{\alpha}$ and $\left.F\right|_{\left\{0, \frac{1}{2}, 1\right\}^{m}}$ can be represented by the mappings $f_{\alpha}$ and $f$, respectively. 
The following relationship between these mappings exists:

$$
f_{\alpha}\left(m_{1}, m_{2}, m_{3}\right)=\left\{\begin{array}{l}
1, \text { if } f\left(m_{1}, m_{2}, m_{3}\right)>\alpha, \\
\frac{1}{2}, \text { if } 1-\alpha \leq f\left(m_{1}, m_{2}, m_{3}\right) \leq \alpha \\
0, \text { if } f\left(m_{1}, m_{2}, m_{3}\right)<1-\alpha .
\end{array}\right.
$$

\subsection{OWA operators}

Yager [29] introduced OWA operators as a tool for aggregation procedures in multicriteria decision making. Usually, OWA operators are defined as functions whose domain is $\mathbb{R}^{m}$. Since in this paper individual intensities of preference vary between 0 and 1 , we have restricted their domain to $[0,1]^{m}$.

Definition 13 Let $\mathbf{w}=\left(w_{1}, \ldots, w_{m}\right) \in[0,1]^{m}$ satisfying $\sum_{i=1}^{m} w_{i}=1$. The $O W A$ operator associated with $\mathbf{w}$ is the aggregation function $F^{\mathbf{w}}$ defined by

$$
F^{\mathbf{w}}(\mathbf{r})=\sum_{i=1}^{m} w_{i} r_{\sigma(i)}
$$

where $\sigma$ is a permutation of $\{1, \ldots, m\}$ such that $r_{\sigma(1)} \geq \cdots \geq r_{\sigma(m)}$.

OWA operators are symmetric and monotonic aggregation functions. Self-dual OWA operators have been characterized by Marichal [16, p. 103] and GarcíaLapresta and Llamazares [8], while characterizations of strictly monotonic OWA operators can be found in Marichal [16, p. 103] and Llamazares [12]. Given $a \in \mathbb{R}$, we denote by $[a]$ the integer part of $a$, i.e., the largest integer smaller than or equal to $a$.

Proposition 14 If $F^{\mathrm{w}}$ is an OWA operator, then:

(1) $F^{\mathbf{w}}$ is self-dual if and only if $w_{m+1-i}=w_{i}$ for all $i \in\left\{1, \ldots,\left[\frac{m}{2}\right]\right\}$.

(2) $F^{\mathrm{w}}$ is strictly monotonic if and only if $w_{i}>0$ for all $i \in\{1, \ldots, m\}$.

In [29], Yager also introduced a measure to characterize the degree to which the aggregation is like an or operation.

Definition 15 The orness measure of an OWA operator $F^{\mathbf{w}}$ is defined by

$$
\operatorname{orness}(\mathbf{w})=\frac{1}{m-1} \sum_{i=1}^{m}(m-i) w_{i}
$$


In order to guarantee an egalitarian treatment of the alternatives, we consider self-dual OWA operators. The weights and the orness of these aggregation operators satisfy the following relationships:

Remark 16 If $F^{\mathrm{w}}$ is a self-dual OWA operator, then:

(1) If $m$ is odd: $2 \sum_{i=1}^{\frac{m-1}{2}} w_{i}+w_{\frac{m+1}{2}}=1$.

(2) If $m$ is even: $2 \sum_{i=1}^{\frac{m}{2}} w_{i}=1$.

(3) $\operatorname{orness}(\mathbf{w})=0.5$.

We will denote by $\mathcal{W}$ the set of weighting vectors associated with self-dual OWA operators, i.e.,

$$
\mathcal{W}=\left\{\mathbf{w} \in[0,1]^{m} \mid \sum_{i=1}^{m} w_{i}=1 \text { and } w_{m+1-i}=w_{i} \text { for all } i \in\left\{1, \ldots,\left[\frac{m}{2}\right]\right\}\right\}
$$

Remark 17 Given an OWA operator $F^{\mathbf{w}}$, the restriction $\left.F^{\mathbf{w}}\right|_{\left\{0, \frac{1}{2}, 1\right\}^{m}}$ is represented by the mapping $f^{\mathbf{w}}$ defined by

$$
f^{\mathbf{w}}\left(m_{1}, m_{2}, m_{3}\right)=\sum_{i=1}^{m_{1}} w_{i}+\frac{1}{2} \sum_{i=1}^{m_{2}} w_{m_{1}+i} .
$$

\subsection{Previous results}

Self-dual OWA operators generalizing simple and absolute special majorities have been characterized by Llamazares [12].

Theorem 18 Let $F^{\mathbf{w}}$ be a self-dual OWA operator and $\alpha \in\left[\frac{1}{2}, 1\right)$. Then the following statements are equivalent:

(1) $F_{\alpha}^{\mathbf{w}}=H_{S}$.

(2) $F^{\mathbf{w}}$ is strictly monotonic and $\alpha<\frac{1}{2}\left(1+\min _{i} w_{i}\right)$.

Theorem 19 Let $F^{\mathbf{w}}$ be a self-dual OWA operator and $\alpha \in\left[\frac{1}{2}, 1\right)$. Then the following statements are equivalent:

(1) $F_{\alpha}^{\mathbf{w}}=H_{A}$.

(2) (a) If $m$ is odd: $w_{\frac{m+1}{2}}>\frac{1}{3}$ and $\frac{3-w_{\frac{m+1}{2}}}{4} \leq \alpha<\frac{1+w_{\frac{m+1}{2}}}{2}$.

(b) If $m$ is even: $w_{\frac{m}{2}}>\frac{1}{4}$ and $\frac{3}{4} \leq \alpha<\frac{1}{2}+w \frac{m}{2}$. 
Theorem 20 Let $F^{\mathbf{w}}$ be a self-dual OWA operator and $\alpha, \beta \in\left[\frac{1}{2}, 1\right)$, with $[\beta m]>\frac{m}{2}$. Then the following statements are equivalent:

(1) $F_{\alpha}^{\mathbf{w}}=Q_{\beta}$.

(2) $w_{m-[\beta m]}>\sum_{i=1}^{m-[\beta m]-1} w_{i}$ and $1-\frac{1}{2} \sum_{i=1}^{m-[\beta m]} w_{i} \leq \alpha<1-\sum_{i=1}^{m-[\beta m]-1} w_{i}$.

As a specific case of this result (for $[\beta m]=m-1$ ), we can obtain a characterization of self-dual OWA operators that generalize unanimous majority.

Corollary 21 Let $F^{\mathbf{w}}$ be a self-dual OWA operator and $\alpha \in\left[\frac{1}{2}, 1\right)$. Then the following statements are equivalent:

(1) $F_{\alpha}^{\mathbf{w}}=H_{U}$.

(2) $w_{1}>0$ and $\alpha \geq 1-\frac{1}{2} w_{1}$.

\section{Generalization of Pareto majority}

In order to generalize Pareto majority by means of self-dual OWA operators, we are going to characterize this majority through two elements of $\mathcal{M}$. The first one corresponds to the minimum support that alternative $x$ needs to be selected. The second corresponds to the maximum support that alternative $x$ can obtain without being selected.

Proposition 22 Let $H$ be a symmetric, monotonic, and self-dual DAF represented by $h$. Then the following statements are equivalent:

(1) $H=H_{P}$.

(2) $h(1, m-1,0)=1$ and $h(m-1,0,1)<1$.

\section{PROOF.}

$(1) \Rightarrow(2)$ : Obvious.

$(2) \Rightarrow(1)$ : Given $\left(m_{1}, m_{2}, m_{3}\right) \in \mathcal{M}$, we distinguish three cases:

(a) If $m_{3}=0$ and $m_{1}>0$, then $\left(m_{1}, m_{2}, m_{3}\right) \succeq(1, m-1,0)$, and, by monotonicity of $H$, we have $h\left(m_{1}, m_{2}, m_{3}\right) \geq h(1, m-1,0)=1$.

(b) If $m_{3}=m_{1}=0$, then by Remark 8 we have $h\left(m_{1}, m_{2}, m_{3}\right)=\frac{1}{2}$.

(c) If $m_{3}>0$, then $(m-1,0,1) \succeq\left(m_{1}, m_{2}, m_{3}\right)$, and, by monotonicity of $H$, we have $h\left(m_{1}, m_{2}, m_{3}\right) \leq h(m-1,0,1)<1$.

Therefore, $h\left(m_{1}, m_{2}, m_{3}\right)=1$ if and only if $m_{1}>0$ and $m_{3}=0$. 
Now we are going to calculate the values that $f^{\mathbf{w}}$ takes in the elements that characterize Pareto majority.

Remark 23 Let $F^{\mathbf{w}}$ be a self-dual OWA operator and $f^{\mathbf{w}}$ be the mapping that represents $\left.F^{\mathbf{w}}\right|_{\left\{0, \frac{1}{2}, 1\right\}^{m}}$. By (1) of Proposition 14 and (1) and (2) of Remark 16 , in the elements $\left(m_{1}, m_{2}, m_{3}\right) \in \mathcal{M}$ that characterize Pareto majority, the mapping $f^{\mathbf{w}}$ takes the following values:

(1) If $m$ is odd:

$$
\begin{aligned}
& f^{\mathbf{w}}(1, m-1,0)=\frac{3}{2} w_{1}+\sum_{i=2}^{\frac{m-1}{2}} w_{i}+\frac{1}{2} w_{\frac{m+1}{2}}=\frac{1}{2}+\frac{1}{2} w_{1}, \\
& f^{\mathbf{w}}(m-1,0,1)=w_{1}+2 \sum_{i=2}^{\frac{m-1}{2}} w_{i}+w_{\frac{m+1}{2}}=1-w_{1} .
\end{aligned}
$$

(2) If $m$ is even:

$$
\begin{aligned}
& f^{\mathbf{w}}(1, m-1,0)=\frac{3}{2} w_{1}+\sum_{i=2}^{\frac{m}{2}} w_{i}=\frac{1}{2}+\frac{1}{2} w_{1}, \\
& f^{\mathbf{w}}(m-1,0,1)=w_{1}+2 \sum_{i=2}^{\frac{m}{2}} w_{i}=1-w_{1} .
\end{aligned}
$$

Therefore, $f^{\mathbf{w}}(1, m-1,0)=\frac{1}{2}\left(1+w_{1}\right)$ and $f^{\mathbf{w}}(m-1,0,1)=1-w_{1}$.

From the previous results we can now characterize the self-dual OWA operators for which the $\alpha$-DAFs associated are Pareto majority.

Theorem 24 Let $F^{\mathbf{w}}$ be a self-dual OWA operator and $\alpha \in\left[\frac{1}{2}, 1\right)$. Then the following statements are equivalent:

(1) $F_{\alpha}^{\mathbf{w}}=H_{P}$.

(2) $w_{1}>\frac{1}{3}$ and $1-w_{1} \leq \alpha<\frac{1}{2}\left(1+w_{1}\right)$.

PROOF. Let $f^{\mathbf{w}}$ be the mapping that represents $\left.F^{\mathbf{w}}\right|_{\left\{0, \frac{1}{2}, 1\right\}^{m}}$. By Proposition 22 and Remark 12 we have that condition $F_{\alpha}^{\mathbf{w}}=H_{P}$ is equivalent to $f^{\mathbf{w}}(1, m-$ $1,0)>\alpha$ and $f^{\mathbf{w}}(m-1,0,1) \leq \alpha$. By Remark 23 we have

$$
F_{\alpha}^{\mathbf{w}}=H_{P} \Leftrightarrow 1-w_{1} \leq \alpha<\frac{1}{2}\left(1+w_{1}\right)
$$


To demonstrate the thesis of the theorem it is sufficient to take into account that

$$
1-w_{1}<\frac{1}{2}\left(1+w_{1}\right) \Leftrightarrow w_{1}>\frac{1}{3} .
$$

\section{Sets of $\alpha$ values and weighting vectors}

In order to obtain a DAF from OWA operators, two parameters must be taken into account: the weighting vector and $\alpha$. Now we analyze some properties of the set containing the valid values of a parameter when we fix the other one.

As we have seen in Theorems 18, 19, 20, and 24, the valid values of $\alpha$ for obtaining a specific majority rule vary between a minimum and a maximum value when the self-dual OWA operator is fixed. This is not a coincidence, but for each self-dual OWA operator $F^{\mathrm{w}}$ and for each symmetric, monotonic, and self-dual DAF $H$, the set $\left\{\alpha \in\left[\frac{1}{2}, 1\right) \mid F_{\alpha}^{\mathbf{w}}=H\right\}$ is an interval (it can be empty).

Definition 25 For each self-dual OWA operator $F^{\mathbf{w}}$, with $\left.F^{\mathbf{w}}\right|_{\left\{0, \frac{1}{2}, 1\right\}^{m}}$ represented by $f^{\mathbf{w}}$, and for each symmetric, monotonic, and self-dual DAF $H$ represented by $h$, we define the following values:

$$
\begin{aligned}
& \underline{\alpha}(\mathbf{w}, H)=\max \left\{f^{\mathbf{w}}\left(m_{1}, m_{2}, m_{3}\right) \mid h\left(m_{1}, m_{2}, m_{3}\right)<1\right\}, \\
& \bar{\alpha}(\mathbf{w}, H)=\min \left\{f^{\mathbf{w}}\left(m_{1}, m_{2}, m_{3}\right) \mid h\left(m_{1}, m_{2}, m_{3}\right)=1\right\},
\end{aligned}
$$

and we denote by $I(\mathbf{w}, H)$ the interval $[\underline{\alpha}(\mathbf{w}, H), \bar{\alpha}(\mathbf{w}, H))$.

Proposition 26 Let $F^{\mathrm{w}}$ be a self-dual OWA operator and $H$ be a symmetric, monotonic, and self-dual DAF. Then the following statements are equivalent:

(1) $F_{\alpha}^{\mathbf{w}}=H$.

(2) $\alpha \in I(\mathbf{w}, H)$.

PROOF. It is obvious from Remark 12 and Definition 25.

From characterization Theorems 18, 19, 20, and 24, the intervals of $\alpha$ values for obtaining simple, absolute, Pareto, and absolute special majorities can be straightforward determined. 
Remark 27 Let $\mathbf{w} \in \mathcal{W}$. Then:

(1) $I\left(\mathbf{w}, H_{S}\right)=\left[\frac{1}{2}, \frac{1}{2}\left(1+\min _{i} w_{i}\right)\right)$, if $w_{i}>0$ for all $i \in\{1, \ldots, m\}$.

(2) (a) $I\left(\mathbf{w}, H_{A}\right)=\left[\frac{1}{4}\left(3-w_{\frac{m+1}{2}}\right), \frac{1}{2}\left(1+w_{\frac{m+1}{2}}\right)\right)$, if $m$ is odd and $w_{\frac{m+1}{2}}>\frac{1}{3}$.

(b) $I\left(\mathbf{w}, H_{A}\right)=\left[\frac{3}{4}, \frac{1}{2}+w_{\frac{m}{2}}\right)$, if $m$ is even and $w_{\frac{m}{2}}>\frac{1}{4}$.

(3) $I\left(\mathbf{w}, Q_{\beta}\right)=\left[1-\frac{1}{2}\left(w_{1}+\cdots+w_{m-[\beta m]}\right), 1-\left(w_{1}+\cdots+w_{m-[\beta m]-1}\right)\right)$, if $[\beta m]>\frac{m}{2}$ and $w_{m-[\beta m]}>w_{1}+\cdots+w_{m-[\beta m]-1}$.

(4) $I\left(\mathbf{w}, H_{P}\right)=\left[1-w_{1}, \frac{1}{2}\left(1+w_{1}\right)\right)$, if $w_{1}>\frac{1}{3}$.

It is worth noting that, given two self-dual OWA operators, the intervals of $\alpha$ values for obtaining a symmetric, monotonic, and self-dual DAF might have empty intersection, as we show in the following example.

Example 28 Consider $m=5$ and $\beta=0.6$. By Theorem $20, F_{\alpha}^{\mathbf{w}}=Q_{\beta}$ if and only if $w_{2}>w_{1}$ and $1-\frac{1}{2}\left(w_{1}+w_{2}\right) \leq \alpha<1-w_{1}$. If we consider $\mathbf{w}=(0.2,0.3,0,0.3,0.2)$, then $I\left(\mathbf{w}, Q_{\beta}\right)=[0.75,0.8)$. However, when we consider the weighting vector $\mathbf{w}^{\prime}=(0.1,0.2,0.2,0.2,0.1)$, we have $I\left(\mathbf{w}^{\prime}, Q_{\beta}\right)=$ $[0.85,0.9)$.

We can obtain a similar result to that of Proposition 26 when we fix the value of $\alpha$. In this case, the set of weighting vectors associated with self-dual OWA operators which allow us to obtain a symmetric, monotonic, and self-dual DAF is convex.

Proposition 29 Let $\alpha \in\left[\frac{1}{2}, 1\right)$ and $H$ be a symmetric, monotonic, and selfdual DAF. Then $\mathcal{W}(\alpha, H)=\left\{\mathbf{w} \in \mathcal{W} \mid F_{\alpha}^{\mathbf{w}}=H\right\}$ is a convex set.

PROOF. Given $p \in[0,1]$ and $\mathbf{w}, \mathbf{w}^{\prime} \in \mathcal{W}(\alpha, H)$, we are going to prove that $\mathbf{w}^{\prime \prime}=p \mathbf{w}+(1-p) \mathbf{w}^{\prime} \in \mathcal{W}(\alpha, H)$. Obviously $\mathbf{w}^{\prime \prime} \in \mathcal{W}$. Let $\mathbf{r} \in\left\{0, \frac{1}{2}, 1\right\}^{m}$, then

$$
\begin{aligned}
F^{\mathbf{w}^{\prime \prime}}(\mathbf{r}) & =\sum_{i=1}^{m}\left(p w_{i}+(1-p) w_{i}^{\prime}\right) r_{\sigma(i)}=p \sum_{i=1}^{m} w_{i} r_{\sigma(i)}+(1-p) \sum_{i=1}^{m} w_{i}^{\prime} r_{\sigma(i)} \\
& =p F^{\mathbf{w}}(\mathbf{r})+(1-p) F^{\mathbf{w}^{\prime}}(\mathbf{r}) .
\end{aligned}
$$

Since $F_{\alpha}^{\mathbf{w}}(\mathbf{r})=F_{\alpha}^{\mathbf{w}^{\prime}}(\mathbf{r})=H(\mathbf{r})$, we distinguish three cases:

(1) If $H(\mathbf{r})=1$, then $\min \left(F^{\mathbf{w}}(\mathbf{r}), F^{\mathbf{w}^{\prime}}(\mathbf{r})\right)>\alpha$. Therefore $F^{\mathbf{w}^{\prime \prime}}(\mathbf{r})>\alpha$, i.e., $F_{\alpha}^{\mathbf{w}^{\prime \prime}}(\mathbf{r})=1$.

(2) If $H(\mathbf{r})=\frac{1}{2}$, then $1-\alpha \leq \min \left(F^{\mathbf{w}}(\mathbf{r}), F^{\mathbf{w}^{\prime}}(\mathbf{r})\right) \leq \max \left(F^{\mathbf{w}}(\mathbf{r}), F^{\mathbf{w}^{\prime}}(\mathbf{r})\right) \leq$ $\alpha$. Therefore $1-\alpha \leq F^{\mathbf{w}^{\prime \prime}}(\mathbf{r}) \leq \alpha$, i.e., $F_{\alpha}^{\mathbf{w}^{\prime \prime}}(\mathbf{r})=\frac{1}{2}$.

(3) If $H(\mathbf{r})=0$, then $\max \left(F^{\mathbf{w}}(\mathbf{r}), F^{\mathbf{w}^{\prime}}(\mathbf{r})\right)<1-\alpha$. Therefore $F^{\mathbf{w}^{\prime \prime}}(\mathbf{r})<1-\alpha$, i.e., $F_{\alpha}^{\mathbf{w}^{\prime \prime}}(\mathbf{r})=0$. 
Therefore $F_{\alpha}^{\mathbf{w}^{\prime \prime}}=H$ and, consequently, $\mathbf{w}^{\prime \prime} \in \mathcal{W}(\alpha, H)$.

\section{Choosing the best-suited weighting vectors}

As we have showed in Theorems 18, 19, 20, 24, and Corollary 21, a lot of selfdual OWA operators that generate the same DAF exist. However, as we will show in the following example, not all of them are suitable for representing a specific majority rule.

Example 30 Consider $m=3, \mathbf{w}=(0.01,0.98,0.01)$ and $\alpha=0.502$. By Theorem $18, F_{\alpha}^{\mathbf{w}}=H_{S}$. However, these weights are close to that of the vector $\mathbf{w}^{\prime}=(0,1,0)$, and, in this case, $F_{\alpha}^{\mathbf{w}^{\prime}}=H_{A}$ for all $\alpha \in\left[\frac{1}{2}, 1\right)$ by Theorem 19 (in fact, with a suitable value of $\alpha$, we would be able to choose the weights of $\mathbf{w}$ as close as necessary to that of $\left.\mathbf{w}^{\prime}\right)$. Therefore, although $F_{\alpha}^{\mathbf{w}}=H_{S}$, the choice of $\mathbf{w}$ and $\alpha$ does not seem the best-suited for representing simple majority.

In order to avoid the previous situation, we propose choosing self-dual OWA operators that maximize the measure of $I(\mathbf{w}, H)$, i.e., the solution of the following problem:

$$
\max _{\mathbf{w} \in \mathcal{W}} \bar{\alpha}(\mathbf{w}, H)-\underline{\alpha}(\mathbf{w}, H)
$$

In the following theorem we show the optimal solutions for simple, absolute, Pareto, and absolute special majorities.

Theorem 31 Let $H$ be a symmetric, monotonic, and self-dual DAF and $\mathbf{w}^{*}$ be a weighting vector solution of problem $(P)$. The following statements hold:

(1) If $H=H_{S}$, then $F^{\mathbf{w}^{*}}$ is the arithmetic mean and $I\left(\mathbf{w}^{*}, H_{S}\right)=\left[\frac{1}{2}, \frac{m+1}{2 m}\right)$.

(2) If $H=H_{A}$, then $F^{\mathbf{w}^{*}}$ is the median. Moreover,

(a) $I\left(\mathbf{w}^{*}, H_{A}\right)=\left[\frac{1}{2}, 1\right)$, if $m$ is odd.

(b) $I\left(\mathbf{w}^{*}, H_{A}\right)=\left[\frac{3}{4}, 1\right)$, if $m$ is even.

(3) If $H=Q_{\beta}$, with $[\beta m]>\frac{m}{2}$, then

$$
\begin{aligned}
& \qquad w_{i}^{*}=\left\{\begin{array}{l}
\frac{1}{2}, \text { if } i=m-[\beta m],[\beta m]+1, \\
0, \text { otherwise, }
\end{array}\right. \\
& \text { and } I\left(\mathbf{w}^{*}, Q_{\beta}\right)=\left[\frac{3}{4}, 1\right) .
\end{aligned}
$$


(4) If $H=H_{P}$, then

$$
\begin{aligned}
& \qquad w_{i}^{*}=\left\{\begin{array}{l}
\frac{1}{2}, \text { if } i=1, m, \\
0, \text { otherwise, }
\end{array}\right. \\
& \text { and } I\left(\mathbf{w}^{*}, H_{P}\right)=\left[\frac{1}{2}, \frac{3}{4}\right) .
\end{aligned}
$$

PROOF. It is obvious from Remark 27.

In Example 28 we have seen that, in general, given a symmetric, monotonic, and self-dual DAF, there is no relationship between the intervals of $\alpha$ values associated with two weighting vectors. However, the weighting vector solution of problem (P) satisfies the following property: given a weighting vector, if a value of $\alpha$ is valid for obtaining a majority rule, then this value is also valid when we consider the weighting vector solution of problem (P). Therefore, for the previously considered majority rules, the self-dual OWA operators given in Theorem 31 are the best-suited.

Proposition 32 Let $H=H_{S}, H=H_{A}, H=Q_{\beta}$ (with $[\beta m]>\frac{m}{2}$ ) or $H=H_{P}$. If $\mathbf{w}^{*}$ is the weighting vector solution of problem $(P)$, then $I(\mathbf{w}, H) \subseteq$ $I\left(\mathbf{w}^{*}, H\right)$ for all $\mathbf{w} \in \mathcal{W}$.

PROOF. It is obvious from Remark 27 and Theorem 31.

The OWA operators obtained in Theorem 31 are the arithmetic mean, the median, and the average of the $j$-th and the $(m+1-j)$-th order statistics. Since they are the best-suited for generating some of the most important classes of majority rules, it would be interesting to know the $\alpha$-DAFs associated with these OWA operators.

As arithmetic mean is a specific case of quasiarithmetic means, we can obtain the $\alpha$-DAFs associated with it from the result given by García-Lapresta and Llamazares [8] for quasiarithmetic means.

Proposition 33 If $F^{\mathbf{w}}$ is the arithmetic mean and $\alpha \in\left[\frac{1}{2}, 1\right)$, then $F_{\alpha}^{\mathbf{w}}$ coincides with the symmetric and self-dual DAF $H$ defined by

$$
h\left(m_{1}, m_{2}, m_{3}\right)=1 \Leftrightarrow m_{1}>m_{3}+m(2 \alpha-1) .
$$

The resultant DAFs are based on difference of votes: an alternative wins when the difference between the number of votes obtained by this alternative and 
that obtained by the other is greater than $m(2 \alpha-1)$. These majority rules have some very interesting features and they have been characterized by Llamazares $[13]$.

In relation to the median, by (2a) of Theorem 31, we have that $F_{\alpha}^{\mathbf{w}}=H_{A}$ for all $\alpha \in\left[\frac{1}{2}, 1\right)$ when $m$ is odd. When $m$ is even, the median is the average of the $m / 2$-th and the $(m / 2+1)$-th order statistics. Therefore, the $\alpha$-DAFs associated with it can be obtained as a specific case of the result given for the average of the $j$-th and the $(m+1-j)$-th order statistics. For analyzing this class of OWA operators we need to know the values taken by $f^{\mathbf{w}}$, which is the mapping that represents $\left.F^{\mathbf{w}}\right|_{\left\{0, \frac{1}{2}, 1\right\}^{m}}$.

Remark 34 Let $j \in\left\{1, \ldots,\left[\frac{m}{2}\right]\right\}$ and $\mathbf{w}$ be the weighting vector defined by

$$
w_{i}=\left\{\begin{array}{l}
\frac{1}{2}, \text { if } i=j, m+1-j, \\
0, \text { otherwise }
\end{array}\right.
$$

Since $F^{\mathbf{w}}(\mathbf{r})=\frac{1}{2}\left(r_{\sigma(j)}+r_{\sigma(m+1-j)}\right)$, then $f^{\mathbf{w}}\left(m_{1}, m_{2}, m_{3}\right)$ takes the following values:

(1) 0 , if $m_{3} \geq m+1-j$.

(2) $\frac{1}{4}$, if $m_{1}<j$ and $j \leq m_{3}<m+1-j$.

(3) $\frac{1}{2}$, if $m_{1}<j$ and $m_{3}<j$, or $m_{1} \geq j$ and $m_{3} \geq j$.

(4) $\frac{3}{4}$, if $j \leq m_{1}<m+1-j$ and $m_{3}<j$.

(5) 1 , if $m_{1} \geq m+1-j$

Proposition 35 Let $j \in\left\{1, \ldots,\left[\frac{m}{2}\right]\right\}$ and $\mathbf{w}$ be the weighting vector defined by

$$
w_{i}=\left\{\begin{array}{l}
\frac{1}{2}, \text { if } i=j, m+1-j \\
0, \text { otherwise }
\end{array}\right.
$$

The following statements are satisfied:

(1) If $\frac{1}{2} \leq \alpha<\frac{3}{4}$, then $F_{\alpha}^{\mathbf{w}}$ coincides with the symmetric and self-dual DAF $H$ defined by

$$
h\left(m_{1}, m_{2}, m_{3}\right)=1 \Leftrightarrow m_{1} \geq j \text { and } m_{3}<j .
$$

(2) If $\frac{3}{4} \leq \alpha<1$, then $F_{\alpha}^{\mathbf{w}}=Q_{\beta}$ for $1-\frac{j}{m} \leq \beta<1-\frac{j-1}{m}$. 
PROOF. Let $f^{\mathbf{w}}$ be the mapping that represents $\left.F^{\mathbf{w}}\right|_{\left\{0, \frac{1}{2}, 1\right\}^{m}}$. Given $\alpha \in$ $\left[\frac{1}{2}, 1\right)$, we have:

(1) If $\frac{1}{2} \leq \alpha<\frac{3}{4}$, then by Remark 34, $f^{\mathbf{w}}\left(m_{1}, m_{2}, m_{3}\right)>\alpha$ if and only if $f^{\mathbf{w}}\left(m_{1}, m_{2}, m_{3}\right) \in\left\{\frac{3}{4}, 1\right\}$; i.e., if and only if $m_{1} \geq j$ and $m_{3}<j$. By Remark 12 we have the result.

(2) If $\frac{3}{4} \leq \alpha<1$, then the result is obvious from items (2b) and (3) of Theorem 31 .

For $j=1$, i.e., when the OWA operator is the average of the maximum and the minimum, it is worth noting that the $\alpha$-DAFs obtained are Pareto and unanimous majorities:

$$
F_{\alpha}^{\mathbf{w}}=\left\{\begin{array}{l}
H_{P}, \text { if } \frac{1}{2} \leq \alpha<\frac{3}{4} \\
H_{U}, \text { if } \frac{3}{4} \leq \alpha<1
\end{array}\right.
$$

\section{Concluding Remarks}

In this paper we have proposed determining OWA operator weights regarding the class of majority rule that we want to obtain when individuals do not grade their preferences between the alternatives. We have also characterized the OWA operators that generalize Pareto majority. Moreover, since the same majority rule can be generalized through a wide variety of OWA operators, we have suggested a procedure to determine the best-suited OWA operators in order to extend simple, Pareto, and absolute special majorities. By means of this procedure we obtain as a remarkable result that the best-suited OWA operators are such well-known aggregation operators as the arithmetic mean, the median, and the average of the $j$-th and the $(m+1-j)$-th order statistics.

\section{Acknowledgements}

This work is partially supported by the Junta de Castilla y León (Consejería de Educación y Cultura, Project VA040A05), the Spanish Ministerio de Educación y Ciencia (Project SEJ2006-04267), and ERDF. The author is grateful to W. Pedrycz and three anonymous referees for valuable comments and references. 


\section{References}

[1] G.R. Amin, A. Emrouznejad, An extended minimax disparity to determine the OWA operator weights, Computers \& Industrial Engineering 50 (2006) 312-316.

[2] T. Calvo, G. Mayor, R. Mesiar (Eds.), Aggregation Operators: New Trends and Applications, Physica-Verlag, Heidelberg, 2002.

[3] D. Filev, R.R. Yager, On the issue of obtaining OWA operator weights, Fuzzy Sets and Systems 94 (1998) 157-169.

[4] R. Fullér, P. Majlender, An analytic approach for obtaining maximal entropy OWA operator weights, Fuzzy Sets and Systems 124 (2001) 53-57.

[5] R. Fullér, P. Majlender, On obtaining minimal variability OWA operator weights, Fuzzy Sets and Systems 136 (2003) 203-215.

[6] J.L. García Lapresta, A general class of simple majority decision rules based on linguistic opinions, Information Sciences 176 (2006) 352-365.

[7] J.L. García Lapresta, B. Llamazares, Aggregation of fuzzy preferences: Some rules of the mean, Social Choice and Welfare 17 (2000) 673-690.

[8] J.L. García Lapresta, B. Llamazares, Majority decisions based on difference of votes, Journal of Mathematical Economics 35 (2001) 463-481.

[9] X. Liu, On the properties of equidifferent OWA operator, International Journal of Approximate Reasoning 43 (2006) 90-107.

[10] X. Liu, L. Chen, On the properties of parametric geometric OWA operator, International Journal of Approximate Reasoning 35 (2004) 163-178.

[11] X. Liu, H. Lou, Parameterized additive neat OWA operators with different orness levels, International Journal of Intelligent Systems 21 (2006) 1045-1072.

[12] B. Llamazares, Simple and absolute special majorities generated by OWA operators, European Journal of Operational Research 158 (2004) 707-720.

[13] B. Llamazares, The forgotten decision rules: Majority rules based on difference of votes, Mathematical Social Sciences 51 (2006) 311-326.

[14] K.C. Maes, S. Saminger, B. De Baets, Representation and construction of selfdual aggregation operators, European Journal of Operational Research 177 (2007) 472-487.

[15] P. Majlender, OWA operators with maximal Rényi entropy, Fuzzy Sets and Systems 155 (2005) 340-360.

[16] J.L. Marichal, Aggregation Operators for Multicriteria Decision Aid, MA Thesis, Liège University, Liège, 1998.

[17] D. Nettleton, V. Torra, A comparison of active set method and genetic algorithm approaches for learning weighting vectors in some aggregation operators, International Journal of Intelligent Systems 16 (2001) 1069-1083. 
[18] M. O'Hagan, Aggregating template or rule antecedents in real-time expert systems with fuzzy set logic, Proc. 22nd Annual IEEE Asilomar Conf. on Signals, Systems and Computers, Pacific Grove, California, 1988, pp. 681-689.

[19] G. Pasi, R.R. Yager, Modeling the concept of majority opinion in group decision making, Information Sciences 176 (2006) 390-414.

[20] J.I. Peláez, J.M. Doña, A majority model in group decision making using QMAOWA operators, International Journal of Intelligent Systems 21 (2006) 193-208.

[21] Y.M. Wang, C. Parkan, A minimax disparity approach for obtaining OWA operator weights, Information Sciences 175 (2005) 20-29.

[22] Y.M. Wang, C. Parkan, Two new approaches for assessing the weights of fuzzy opinions in group decision analysis, Information Sciences 176 (2006) 3538-3555.

[23] Y.M. Wang, C. Parkan, A preemptive goal programming method for aggregating OWA operator weights in group decision making, Information Sciences 177 (2007) 1867-1877.

[24] Z.S. Xu, A method based on linguistic aggregation operators for group decision making with linguistic preference relations, Information Sciences 166 (2004) $19-30$.

[25] Z.S. Xu, An overview of methods for determining OWA weights, International Journal of Intelligent Systems 20 (2005) 843-865.

[26] Z.S. Xu, Dependent OWA operators, in: V. Torra, Y. Narukawa, A. Valls, J. Domingo-Ferrer (Eds.), Modeling Decisions for Artificial Intelligence: Third International Conference MDAI 2006, Lecture Notes in Artificial Intelligence 3885 , Springer, Berlin, 2006, pp. 172-178.

[27] Z.S. Xu, Q.L. Da, The uncertain OWA operator, International Journal of Intelligent Systems 17 (2002) 569-575.

[28] Z.S. Xu, Q.L. Da, An overview of operators for aggregating information, International Journal of Intelligent Systems 18 (2003) 953-969.

[29] R.R. Yager, On ordered weighted averaging operators in multicriteria decision making, IEEE Transactions on Systems, Man and Cybernetics 18 (1988) 183190.

[30] R.R. Yager, Families of OWA operators, Fuzzy Sets and Systems 59 (1993) $125-148$.

[31] R.R. Yager, Quantifier guided aggregation using OWA operators, International Journal of Intelligent Systems 11 (1996) 49-73.

[32] R.R. Yager, E-Z OWA weights, Proc. 10th International Fuzzy Systems Association World Congress, Istanbul, 2003, pp. 39-42.

[33] R.R. Yager, Extending multicriteria decision making by mixing t-norms and OWA operators, International Journal of Intelligent Systems 20 (2005) 453474 . 
[34] R.R. Yager, Centered OWA operators, Soft Computing, in press.

[35] R.R. Yager, D. Filev, Induced ordered weighted averaging operators, IEEE Transactions on Systems, Man and Cybernetics - Part B: Cybernetics 29 (1999) 141-150.

[36] R.R. Yager, J. Kacprzyk (Eds.), The Ordered Weighted Averaging Operators: Theory and Applications, Kluwer Academic Publishers, Dordrecht, 1997. 